\title{
van der Waals interactions between molecular hydrogen and alkali-metal atoms
}

\section{Citation}

Zhu, C., A. Dalgarno, and A. Derevianko. 2002. "Van Der Waals Interactions between Molecular Hydrogen and Alkali-Metal Atoms." Physical Review A 65 (3). https://doi.org/10.1103/ physreva.65.034708.

\section{Permanent link}

http://nrs.harvard.edu/urn-3:HUL.InstRepos:41417412

\section{Terms of Use}

This article was downloaded from Harvard University's DASH repository, and is made available under the terms and conditions applicable to Other Posted Material, as set forth at http:// nrs.harvard.edu/urn-3:HUL.InstRepos:dash.current.terms-of-use\#LAA

\section{Share Your Story}

The Harvard community has made this article openly available.

Please share how this access benefits you. Submit a story.

Accessibility 


\title{
van der Waals interactions between molecular hydrogen and alkali-metal atoms
}

\author{
C. Zhu, ${ }^{1}$ A. Dalgarno, ${ }^{1}$ and A. Derevianko ${ }^{2}$ \\ ${ }^{1}$ ITAMP, Harvard-Smithsonian Center for Astrophysics, 60 Garden Street, Cambridge, Massachusetts 02138 \\ ${ }^{2}$ Department of Physics, University of Nevada, Reno, Nevada 89557
}

(Received 16 October 2001; published 28 February 2002)

\begin{abstract}
In this paper, we have calculated the values of the $\mathrm{C}_{6}$ coefficients and the anisotropy parameters $\gamma$ of the leading term of the van der Waals interactions between molecular hydrogen and alkali-metal atoms from Li to Fr. The uncertainties in our results are less than $2 \%$.
\end{abstract}

DOI: $10.1103 /$ PhysRevA.65.034708

PACS number(s): $34.20 . \mathrm{Gj}$

\section{INTRODUCTION}

Collisions at ultralow temperatures are sensitive to longrange interactions between the colliding species. Of particular importance in the construction of the long-range potential are the $\mathrm{C}_{6}$ coefficient and the anisotropy parameter $\gamma$ of the leading term of the van der Waals interaction, which decreases with nuclear separation $R$ as $R^{-6}$. These two constants can be obtained by integration over the frequency $\omega$ of the product of the dynamic dipole polarizabilities $\alpha(i \omega)$ at imaginary frequencies of the two colliding species. If the collision involves a diatomic molecule, both the parallel and perpendicular components of the dynamic polarizability, $\alpha_{\|}(i \omega)$ and $\alpha_{\perp}(i \omega)$, need to be calculated.

Accurate values of $\alpha_{\|}(i \omega)$ and $\alpha_{\perp}(i \omega)$ for $\mathrm{H}_{2}$ over a range of internuclear distances have been calculated by Ford and Browne [1] using a variational procedure [2]. They obtained $\overline{\mathrm{C}}_{6}=12.09$ a.u. as the average of the $\mathrm{H}_{2}-\mathrm{H}_{2}$ interaction $\mathrm{C}_{6}$ coefficients over all the molecular orientations and all the internuclear distances in the lowest $v=0$ vibrational level. In a similar calculation at the equilibrium separation of the $v=0$ level at $R=1.449 a_{0}$, Bishop and Pipin [3] obtained $\overline{\mathrm{C}}_{6}=12.06$ a.u. These results agree closely with the empirical values $[4,5]$. Thus there is little loss of accuracy in avoiding the vibrational averaging and using the dynamic polarizabilities evaluated at the equilibrium separation. Convenient representations of $\alpha_{\|}(i \omega)$ and $\alpha_{\perp}(i \omega)$ at $R=1.449 a_{0}$ have been constructed by Bishop and Pipin [3]. The possible error in the resulting values of $\mathrm{C}_{6}$ is less than $1 \%$.

Comparable high accuracy has been obtained for the dynamic polarizabilities of $\mathrm{Li}$ [6], and $\mathrm{Na}, \mathrm{K}, \mathrm{Rb}, \mathrm{Cs}$, and $\mathrm{Fr}$ [7]. The polarizabilities of $\mathrm{Na}, \mathrm{K}, \mathrm{Rb}, \mathrm{Cs}$, and $\mathrm{Fr}$ were obtained by an approximation of many-body perturbation theory [7].

\section{CAlCUlations}

The leading term of the interaction energy at a large distance $R$ between a spherical atom and a homonuclear diatomic molecule has the form

$$
V(R)=-\frac{\mathrm{C}_{6}}{R^{6}}\left[1+\gamma P_{2}(\cos \theta)\right],
$$

where $P_{2}(\cos \theta)$ is the second-order Legendre polynomial and $\theta$ is the angle between the symmetry axis of the mol- ecule and the vector from the nucleus of the atom to the center of the molecule. The $\mathrm{C}_{6}$ coefficient and anisotropy parameter $\gamma$ can be expressed as

$$
\mathrm{C}_{6}=\frac{1}{\pi}\left\{\int_{0}^{\infty} \alpha_{\|}(i \omega) \alpha(i \omega) d \omega+2 \int_{0}^{\infty} \alpha_{\perp}(i \omega) \alpha(i \omega) d \omega\right\}
$$

and

$$
\gamma=\frac{1}{\pi \mathrm{C}_{6}}\left\{\int_{0}^{\infty} \alpha_{\|}(i \omega) \alpha(i \omega) d \omega-\int_{0}^{\infty} \alpha_{\perp}(i \omega) \alpha(i \omega) d \omega\right\},
$$

where $\alpha_{\|}(i \omega)$ and $\alpha_{\perp}(i \omega)$ are, respectively, the parallel and the perpendicular dynamic dipole polarizabilities of $\mathrm{H}_{2}$ and $\alpha(i \omega)$ is the dynamic dipole polarizability of an alkali-metal atom. We have plotted in Fig. $1 \alpha_{\|}(i \omega)$ and $\alpha_{\perp}(i \omega)$ for $\mathrm{H}_{2}$, and $\alpha(i \omega)$ for $\mathrm{Na}$ as functions of the frequency $\omega$, as an illustration.

The calculated values of $\mathrm{C}_{6}$ and $\gamma$ are compared in Table I with previous results of Victor and Dalgarno [4]. The values of $\gamma$ agree closely. The differences in $\mathrm{C}_{6}$ range from 0.3

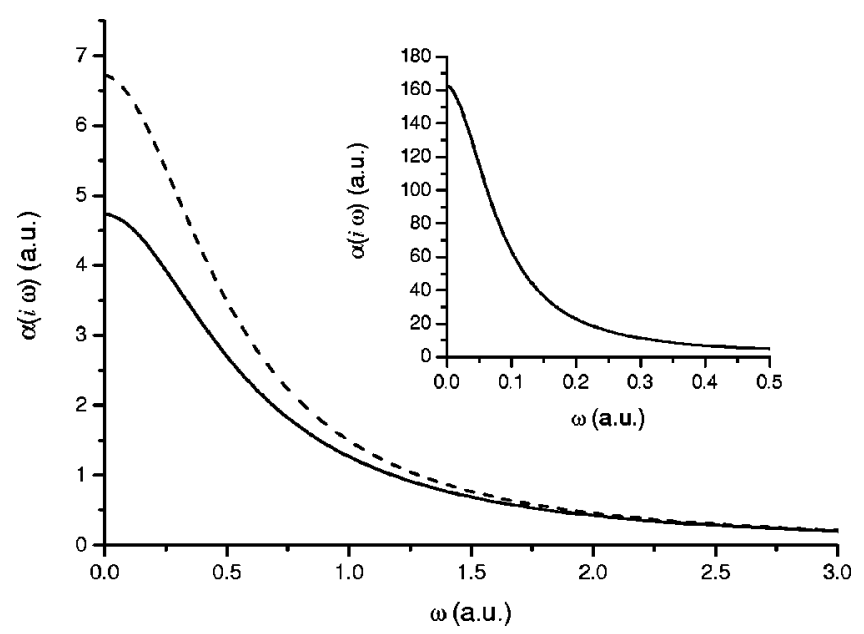

FIG. 1. Dynamic dipole polarizabilities of $\mathrm{H}_{2}$ and $\mathrm{Na}$ as functions of the imaginary frequency $\omega$. The solid and dashed curves denote the perpendicular and parallel components of the dynamic dipole polarizability of $\mathrm{H}_{2}, \alpha_{\perp}(i \omega)$ and $\alpha_{\|}(i \omega)$, respectively. The curve in the inset denotes the dynamic dipole polarizability of $\mathrm{Na}$, $\alpha(i \omega)$. 
TABLE I. $\mathrm{C}_{6}$ coefficients and anisotropy parameters $\gamma$ for interactions between molecular hydrogen and alkali-metal atoms.

\begin{tabular}{ccccc}
\hline \hline & & & & \\
Atom & $\mathrm{C}_{6}[4]$ & $\mathrm{C}_{6}$ (this paper) & $\gamma[4]$ & $\gamma$ (this paper) \\
\hline $\mathrm{Li}$ & 83.5 & 82.6 & 0.118 & 0.117 \\
$\mathrm{Na}$ & 92.0 & 92.3 & 0.118 & 0.116 \\
$\mathrm{~K}$ & 127 & 139 & 0.120 & 0.116 \\
$\mathrm{Rb}$ & 136 & 156 & 0.120 & 0.115 \\
$\mathrm{Cs}$ & 160 & 189 & 0.120 & 0.114 \\
$\mathrm{Fr}$ & & 181 & & 0.113 \\
\hline
\end{tabular}

for $\mathrm{Na}$ to 29 for $\mathrm{Cs}$, reflecting the varying importance of the contribution, omitted by Victor and Dalgarno [4], of core transitions $[8,7]$. The values presented here are limited in accuracy because the method we have used does not allow for the dependence of $\mathrm{C}_{6}$ and $\gamma$ on the rotational or vibrational population of the $\mathrm{H}_{2}$ molecule. An estimate of the uncertainty in $\mathrm{C}_{6}$ can be obtained from the calculations of Kolos and Wolniewicz [9] and of Ford and Browne [1] of the static dipole polarizability of $\mathrm{H}_{2}$ for rotational levels $J$ $=0-4$ of the $v=0$ vibrational level. The corresponding probable uncertainties for $\mathrm{H}_{2}$ in the $v=0$ level interacting with the alkali-metal atoms are less than $2 \%$ for $\mathrm{C}_{6}$ and still smaller for $\gamma$.

\section{ACKNOWLEDGMENTS}

This work was supported by the Chemical Sciences, Geosciences and Biosciences Division of the Office of Basic Energy Sciences, Office of Science, U.S. Department of Energy. The work of A.D. was supported in part by the National Science Foundation. We are grateful to Dr. A. L. Ford for the information about his calculations.
[1] A. L. Ford and J. C. Browne, Phys. Rev. A 7, 418 (1973).

[2] A. Dalgarno, A. L. Ford, and J. C. Browne, Phys. Rev. Lett. 27, 1033 (1971).

[3] D. M. Bishop and J. Pipin, J. Chem. Phys. 97, 3375 (1992).

[4] G. A. Victor and A. Dalgarno, J. Chem. Phys. 53, 1316 (1970).

[5] G. D. Zeiss and W. J. Meath, Mol. Phys. 33, 1155 (1977).
[6] Z.-C. Yan, A. Dalgarno, and J. F. Babb, Phys. Rev. A 55, 2882 (1997).

[7] A. Derevianko, W. R. Johnson, M. S. Safronova, and J. F. Babb, Phys. Rev. Lett. 82, 3589 (1999).

[8] A. Dalgarno and W. D. Davison, Mol. Phys. 13, 479 (1967).

[9] W. Kolos and L. Wolniewicz, J. Chem. Phys. 46, 1426 (1967). 